\title{
Cocaine exposure shifts the balance of associative encoding from ventral to dorsolateral striatum
}

\author{
Yuji Takahashi ${ }^{1 *}$, Matthew R Roesch ${ }^{1}$, Thomas A Stalnaker ${ }^{1}$ and Geoffrey Schoenbaum ${ }^{1,2,3}$ \\ 1. Department of Anatomy and Neurobiology, University of Maryland School of Medicine, USA \\ 2. Department of Psychiatry, University of Maryland School of Medicine, USA \\ 3. Department of Psychology, University of Maryland Baltimore County, USA \\ Edited by: Sidney A. Simon, Duke University, USA \\ Reviewed by: Rui M. Costa, National Institutres of Health, USA \\ Jennifer Stapleton, Duke University, USA
}

\begin{abstract}
Both dorsal and ventral striatum are implicated in the "habitization" of behavior that occurs in addiction. Here we examined the effect of cocaine exposure on associative encoding in these two regions. Neural activity was recorded during go/no-go discrimination learning and reversal. Activity in ventral striatum developed and reversed rapidly, tracking the valence of the predicted outcome, whereas activity in dorsolateral striatum developed and reversed more slowly, tracking discriminative responding. This difference is consistent with the putative roles of these two areas in promoting habit-like behavior. Dorsolateral striatum has been directly implicated in habit or stimulusresponse learning, whereas ventral striatum appears to be involved indirectly by allowing cues associated with reward to exert a general motivational influence on responding. Interestingly cocaine exposure did not uniformly enhance processing across both regions. Instead cocaine reduced the degree and flexibility of cue-evoked firing in ventral striatum while marginally enhanced cue-selective firing in dorsolateral striatum. Thus cocaine exposure causes regionally specific effects on neural processing in striatum; these effects may promote the habitization of behavior by shifting control from ventral to dorsolateral regions.
\end{abstract}

Keywords: nucleus accumbens core, dorsolateral striatum, psychostimulant, decision making, rat, learning

\section{INTRODUCTION}

Exposure to psychostimulants has enduring effects on brain areas that are proposed to promote habitual behavior. These changes have been hypothesized to lead to the loss of control that characterizes drug addiction. Drug-induced changes within the striatum, an area critical to habit learning, may be particularly important for this transition (Everitt and Robbins, 2005; Everitt and Wolf, 2002; Kalivas and Volkow, 2005). The dorsal striatum (DS) has been implicated in motor habits, mediated by stimulus-response associations (Frank and Claus, 2006; Yin and Knowlton, 2006). Neurons in DS fire differently as stimulus-response associations are acquired (Jog et al., 1999; Schmitzer-Torbert and Redish, 2004), and instrumental responding in rats with lesions of lateral DS remains sensitive to outcome devaluation even after prolonged training (Yin et al., 2004). The ventral striatum (VS) has been implicated more

\footnotetext{
* Correspondence: Yuji Takahashi, Department of Anatomy and Neurobiology, University of Maryland School of Medicine, 20 Penn St, HSF-2, S251, Baltimore, Maryland 21201, USA. e-mail: ytaka001@umaryland.edu

Yuji Takahashi and Matthew R Roesch contributed equally to this work. Received: 29 0ct. 2007; paper pending published: 07 Dec. 2007; accepted: 08 Dec. 2007; Published online: 30 Dec. 2007

Full citation: Frontiers in Integrative Neuroscience (2007) 1:11 doi: 10.3389/neuro.07/ 011.2007

Copyright (C) 2007 Takahashi, Roesch, Stalnaker and Schoenbaum. This is an openaccess article subject to an exclusive license agreement between the authors and the Frontiers Research Foundation, which permits unrestricted use, distribution, and reproduction in any medium, provided the original authors and source are credited.
}

indirectly in driving instrumental responding through its role in allowing cues associated with reward to exert a general motivational influence on habits. Neurons in VS-particularly within the accumbens core-fire in response to such reward-associated cues that have acquired value through pairing with reward (Carelli, 2002; Shidara et al., 1998; Schultz et al., 2000; Williams et al., 1993), and damage within this region affects Pavlovian-to-instrumental transfer (Corbit et al., 2001; Hall et al., 2001). Notably instrumental learning in rats previously sensitized to amphetamine becomes resistant to outcome devaluation even without overtraining (Nelson and Killcross, 2006), and it is also more sensitive to the motivating effects of Pavlovian cues in the transfer task (Wyvell and Berridge, 2001). These effects could reflect a generalized drug-induced increase in the efficacy of neural processing in these striatal regions.

To test this hypothesis, we recorded single-unit activity in VS and DS in rats previously exposed to cocaine. Neurons were recorded as rats learned and reversed odor discrimination problems in which unique odor cues were paired with a sucrose reward and a quinine punishment (Schoenbaum et al., 1999). The rats had to attend to the associations between the odor cues and the rewarding and aversive outcomes, presumably resulting in the attribution of motivational value or significance to the cues. In addition, the rats were required to associate each cue with a different response. We found that although neurons in both regions fired to the cues based on their associations with these subsequent events (responses and outcomes), activity in VS emerged before differential responding, whereas activity in DS emerged later, only after differential responding was established. Contrary to predictions, cocaine reduced the degree and flexibility of cue-selective firing in VS while marginally enhancing cue-selective firing in lateral DS. This finding 
suggests that cocaine exposure causes regionally specific effects on neural processing in striatum; these effects may promote the habitization of behavior by shifting control from ventral to dorsolateral regions.

\section{MATERIALS AND METHODS \\ Animals}

Twenty-two Male Long-Evans rats (175-200 g; Charles River Labs, Wilmington, MA) were tested at the University of Maryland School of Medicine in accordance with university and $\mathrm{NIH}$ guidelines.

\section{Cocaine exposure}

Cocaine sensitization was conducted as in previous experiments (Burke et al., 2006; Schoenbaum and Setlow, 2005; Schoenbaum et al., 2004; Stalnaker et al., 2006; Stalnaker et al., 2007a). Briefly, beginning approximately 6 weeks before recording, rats received daily i.p. injections of cocaine $\mathrm{HCl}$ or saline vehicle (NIDA, Bethesda, MD) for 14 days. Locomotor activity was monitored for 1 hour after each injection using overhead activity monitors mounted in clear Plexiglas training chambers (Coulbourn Instruments). The day before the start of the treatment regimen, the rats were placed into the activity chambers for 1 hour to monitor the baseline activity levels. Then rats were divided into two groups with similar activity levels. Over the next 14 days, one group $(n=9)$ received daily i.p. injections of $30 \mathrm{mg} / \mathrm{kg}$ cocaine $\mathrm{HC}(20 \mathrm{mg} / \mathrm{ml})$; the other $(n=13)$ received similar volume injections of $0.9 \%$ saline. At the end of the recording study, brains were removed and processed to allow post-mortem analyses of protein expression and dendritic structure in several brain areas. As a result, we did not re-expose these rats to cocaine at the end of the experiment to confirm the presence of sensitization. However, we have shown in several previous reports, including a comparable recording study, that rats with an identical history of cocaine exposure exhibit evidence of sensitization after similar training (Schoenbaum and Setlow, 2005; Schoenbaum et al., 2004; Setlow et al., 2003; Stalnaker et al., 2007b).

\section{Surgery and histology}

Using aseptic, stereotaxic surgical techniques, a driveable bundle of ten $25 \mu \mathrm{m}$ diameter FeNiCr wires (Stablohm 675, California Fine Wire, Grover Beach, CA) was chronically implanted in the left hemisphere in DS of 11 rats $(0.5 \mathrm{~mm}$ posterior to bregma, $3.6 \mathrm{~mm}$ lateral, and $3.0 \mathrm{~mm}$ ventral) and VS of 11 rats $(1.6 \mathrm{~mm}$ anterior to bregma, $1.5 \mathrm{~mm}$ laterally, and $4.5 \mathrm{~mm}$ ventral). Immediately prior to implantation, these wires were freshly cut with surgical scissors to extend $\sim 1 \mathrm{~mm}$ beyond the cannula and electroplated with platinum $\left(\mathrm{H}_{2} \mathrm{PtCl}_{6}\right.$, Aldrich, Milwaukee, WI) to an impedance of $\sim 300 \mathrm{kOhms}$. At the end of the study, the final electrode position was marked by the passage of a $15 \mu \mathrm{A}$ current through each microwire for 10 seconds to create a small iron deposit. The rats were then perfused with formaldehyde and potassium ferrocyanide solution to visualize the iron deposit. The brains were removed from the skulls and processed for histology using standard techniques (Schoenbaum et al., 1999).

\section{Odor discrimination training}

Discrimination training was conducted in aluminum chambers approximately 18 " on each side with sloping walls narrowing to an area of 12 " $\times 12$ " at the bottom. An odor port and fluid well were located on a panel in the right wall of each chamber below two panel lights; the odor port was connected to an air flow dilution olfactometer to allow the rapid delivery of olfactory cues. Task control was implemented via computer. Odor discrimination problems were composed of odor pairs chosen from compounds obtained from International Flavors and Fragrances (New York, NY). During training, rats were maintained on water restriction for 15-20 minutes/day. Trials were signaled by illumination of the panel lights inside the box. When these lights were on, nosepoke into the odor port resulted in delivery of the pre-selected odor cue to a small hemicylinder located behind this opening. The rat terminated odor sampling by leaving the odor port then had 3 seconds to make a go response at the fluid well located below the port. If a response was made after sampling a positive odor, then a $0.05 \mathrm{ml}$ bolus of a $10 \%$ sucrose solution was delivered to the well after a variable delay $(500-1500 \mathrm{~ms})$. If the same response was made after sampling a negative odor, then a $0.05 \mathrm{ml}$ bolus of a $0.02 \mathrm{M}$ quinine solution was delivered after a similar delay. If the rat did not respond within 3 seconds, the trial was counted as a no-go. Rats typically began each session with a new odor pair by responding on every trial, and then learned to withhold responding after sampling the negative odor. Rats rarely failed to respond on positive trials. A behavioral criterion was defined as 18 correct responses (go on positive trials, no-go on negative trials) in a moving block of 20 trials. Rats were trained for $60-100$ trials after meeting this criterion; then the odor-outcome associations were reversed. Training continued until the rats met criterion on the reversal. Performance phases were defined based on go, no-go performance to include a pre-criterion phase, before the rat met criterion on the discrimination, a post-criterion phase, after the rat met criterion, and a reversal phase, after the odor-outcome associations were switched. The rats received training on several problems prior to surgery, and then neural data were collected as the rats acquired novel discriminations in sessions after surgery.

\section{Single-unit recording}

For each recording session, the rat was placed in the training chamber, and the electrode wires were screened for neural activity while the rat explored the open chamber. Active wires were selected for recording, and a training session was begun. If no activity was detected, the rat was removed, and the electrode assembly was advanced 40 or $80 \mu \mathrm{m}$. Otherwise the electrode was advanced at the end of the session, so that neural activity was never acquired from the same location twice. Neural activity was recorded using two identical Plexon Multichannel Acquisition Processor systems (Dallas, TX), interfaced with odor discrimination training chambers described above. Signals from the electrode wires were amplified 20X by an op-amp headstage (Plexon Inc., HST/8050-G20-GR), located on the electrode array. Immediately outside the training chamber, the signals were passed through a differential pre-amplifier (Plexon Inc., PBX2/16sp-r-G50/16fp-G50), where the single-unit signals were amplified $50 \mathrm{X}$ and filtered at $150-9000 \mathrm{~Hz}$. The single-unit signals were then sent to the Multichannel Acquisition Processor box, where they were further filtered at $250-8000 \mathrm{~Hz}$, digitized at $40 \mathrm{kHz}$ and amplified at 1$32 \mathrm{X}$. Waveforms ( $>2.5: 1$ signal-to-noise) were extracted from active channels and recorded to disk by an associated workstation with event timestamps from the behavior computer. In the present study, 148 neurons were recorded from VS of seven saline-treated rats and 62 neurons from VS of four cocaine-treated rats. These neurons were recorded in the core region of the nucleus accumbens. In lateral DS, 330 neurons were recorded from six saline-treated rats and 426 neurons from five cocaine-treated rats.

\section{Statistical data analysis}

Units were sorted using Offline Sorter software from Plexon Inc. (Dallas, $T X$ ), using a template matching algorithm and notes regarding the waveforms made during the session. Sorted files were then processed in Neuroexplorer to extract unit timestamps and relevant event markers. These data were subsequently analyzed using statistical routines in Matlab (Natick, MA) to examine activity during odor sampling (from $50 \mathrm{~ms}$ after odor onset to $50 \mathrm{~ms}$ after odor offset). Firing activity (spikes/second) in each time window was compared on positive and negative trials during pre- and post-criterion trial blocks and after reversal using ANOVA $(p<0.05)$, and neurons with a significant difference in activity were categorized as "selective" in trial block. A Pearson $X_{2}$ test $(p<0.05)$ was used to compare the proportions of neurons with different firing properties 
in saline- and cocaine-treated rats. In addition, we computed an odorselectivity index for each neuron. This index was calculated as $\left(\mathrm{fr}_{\text {odor1 } 1}-\mathrm{fr}_{\text {odor } 2}\right) /\left(\mathrm{fr}_{\text {odor1 }}+\mathrm{fr}_{\text {odor } 2}\right)$, where $\mathrm{fr}_{\text {odor1 } 1}$ was the firing rate during sampling of the odor that predicted the sucrose outcome during prereversal trials and the odor that predicted the quinine outcome after reversal for a given neuron. $\mathrm{fr}_{\text {odor2 }}$ was the firing rate during sampling of the odor that predicted the quinine outcome during pre-reversal trials and the odor that predicted the sucrose outcome after reversal. Correlation coefficients were calculated to compare this measure before and after reversal in different populations $(p<0.05)$. Finally population histograms were constructed. Firing rate of each neuron was normalized and then averaged across histograms in which neural activity was aligned with odor onset.

\section{RESULTS}

Rats exposed to cocaine exhibited greater locomotor activity than salinetreated controls during drug exposure; consistent with this, a 2-factor ANOVA (group $X$ day) revealed a significant interaction $\left(F_{13,234}=4.16\right.$, $p=0.000003)$, and significant main effects of group $\left(F_{1,18}=23.24\right.$, $p=0.0001)$, and day $\left(F_{13,234}=2.37, p=0.005\right)$. Recording began 4 weeks after the end of drug treatment and continued for approximately 8 weeks. Recording was conducted in a different room and style of training chamber that was used for drug-exposure.

Recording data were taken from sessions in which the rats learned the novel problem and successfully acquired a reversal of that problem, meeting a criterion of 18 correct responses in a moving block of 20 trials both before and again after reversal. Performance in these experimenterselected recording sessions did not differ between groups. Saline-treated rats required $72.2 \pm 3.1$ (mean $\pm \mathrm{SEM}$ ) trials to meet criterion on the initial discriminations and $66.3 \pm 4.1$ trials to meet criterion on the reversals, whereas cocaine-treated rats required $70.0 \pm 3.8$ to learn and $68.3 \pm 5.1$ trials to reverse the discriminations. A two-factor ANOVA (group $X$ learning/reversal) revealed neither main effects nor any interactions $(F<0.64, p>0.64)$. However, the cocaine-treated rats did exhibit a general impairment at reversal learning in that they successfully reversed a much lower proportion of the odor problems to which they were exposed than the saline-treated controls. Thus the cocaine-treated rats met criterion after reversal in only $84 / 130$ possible sessions, whereas the saline-treated rats met criterion in $151 / 188$ sessions $\left(X_{2}=9.83\right.$, $p=0.0017)$. This difference was present even though the cocainetreated rats completed more trials after reversal, on average, than the controls in these unsuccessful sessions $(91.7 \pm 9.4$ and $71.6 \pm 10.3$ trials, respectively). However, there was no significant difference between subjects in both saline- and cocaine-treated groups in terms of behavioral performance (ANOVA, $F<0.79, p>0.65$ ). Thus, as in prior studies, the cocaine-treated rats had more difficulty than controls in reversing the discriminations (Schoenbaum et al., 2004; Stalnaker et al., 2006; Stalnaker et al., 2007b).

Neural activity was recorded using a drivable bundle of microwires. This bundle was advanced between recording sessions, so that data from new neurons could be acquired in each session. Figure 1A shows recording locations. In VS, we recorded 148 neurons in 64 sessions in saline-treated rats $(n=7)$ and 62 neurons in 25 sessions in cocainetreated rats $(n=4)$. These neurons were recorded in the core region of the nucleus accumbens. In lateral DS, we recorded 330 neurons in 81 sessions in saline-treated rats $(n=6)$ and 426 neurons in 58 sessions in cocaine-treated rats $(n=5)$. The distribution of average firing rates for neurons in each group is shown in Figure 1B. Interestingly, although the recording locations were similar between groups within each region, the average firing rate for neurons in the cocaine-treated rats was significantly higher in both VS $(2.64 \mathrm{~s} / \mathrm{s}$ for saline vs. $4.58 \mathrm{~s} / \mathrm{s}$ for cocaine, ANOVA, $\left.F_{1,231}=11.28, p=0.0009\right)$ and DS $(5.56 \mathrm{~s} / \mathrm{s}$ for saline vs. $8.11 \mathrm{~s} / \mathrm{s}$ for cocaine, ANOVA, $F_{1,754}=7.83, p=0.0053$ ). The higher average firing rate was attributable in each case to an increase in the number of very fast spiking neurons in the cocaine-treated rats; this is evident in the distributions in Figure 1B $(>20 \mathrm{~Hz})$. We observed a similar subpopulation in cocaine-treated rats in a recent study in $\mathrm{ABL}$ (Stalnaker et al., 2007b), and elevated baseline firing has also been reported in OFC by another group after exposure to psychostimulants (Homayoun and Moghaddam, 2006). Although the behavioral correlates of these neurons did not appear unique, the number was too small to allow a quantitative comparison. For consistency, we elected to exclude this specific population from the subsequent analyses. This resulted in the final populations shown in Table 1 for each brain region. Note however that their exclusion (or inclusion) did not alter any of the significant results, we will report next.

\section{Cue-selective activity in VS during odor discrimination learning and reversal}

Cue-selective activity is a prominent characteristic of neural activity recorded in VS in awake, behaving animals (Carelli, 2002; Nicola et al., 2004; O'Doherty et al., 2004; Roitman et al., 2005; Setlow et al., 2003). This activity is sensitive to associations between cues and biologically meaningful outcomes, consistent with the proposed role for VS in mediating behaviors that reflect the motivational value of Pavlovian cues. Accordingly, we found that $30 \%$ (44 out of 148) of the VS neurons recorded in saline-treated rats fired differentially as the rats sampled the odor cue after learning (Figure 2 and Table 1). It is important to emphasize that the cue-sampling period preceded both the discriminative response and reward delivery and that the rats were required to remain stationary within the odor port during cue-sampling. Thus differential neural activity during the cue-sampling period is unlikely to be directly caused by either the response or later delivery of reward, though it may reflect these subsequent events due to their associations with the odor cues.

A majority of the cue-selective VS neurons (89\%; 39/44) fired more strongly to the cue predictive of quinine (Figure 2 and Table 1). Indeed, the population of VS neurons that fired more strongly for the positive odor cue was not above chance $\left(X_{2}=0.35, p=0.56\right)$. There was no significant difference between subjects in terms of the proportion of positive, negative cue-selective and non-selective neurons (ANOVA, $F_{2,5}=0.76$, $p=0.60$ ). The importance of the outcome in these cue-selective responses was evident after reversal when most of the cue-selective neurons switched cue preference (Figure 2 and Table 1). These proportions, including the predominance of neurons selective for the quinine-predicting cue, are similar to what we have reported previously in VS in rats learning odor discriminations and reversals (Setlow et al., 2003).

The influence of predicted outcome on cue-selectivity in VS, particularly for the aversive outcome, is also evident in the population responses shown in Figure 3A. These population responses-shown separately for all neurons and for cue-selective neurons only-exhibited a strong phasic response to the negative odor cue during learning and after reversal. The cue-selectivity of each VS neuron is illustrated on a unit-byunit basis in Figure 3B. This figure plots the odor-selectivity indices of each neuron before and after reversal (see Materials and Methods). These indices exhibited a highly significant inverse correlation, indicating that the neurons fired to the predicted outcome with little regard to the cues' sensory features.

Interestingly VS neurons typically developed cue-selective activity before rats acquired behavioral criterion. This was true both before and after reversal. Thus of the 39 vS neurons that were selective for the negative odor cue, more than a half of them (22 out of 39) developed this selective response in late part of the pre-criterion trial block (Figure 2 and Table 1). The influence of these neurons is evident in the population response, which exhibited a phasic response to the negative odor cue even during the pre-criterion trials (Figure 3A). Similarly after reversal, most (18 out of 24) of these VS neurons that reversed their cue-selectivity did so before the rats met criterion on the reversed discrimination (Figure 2 and Table 1). 
A
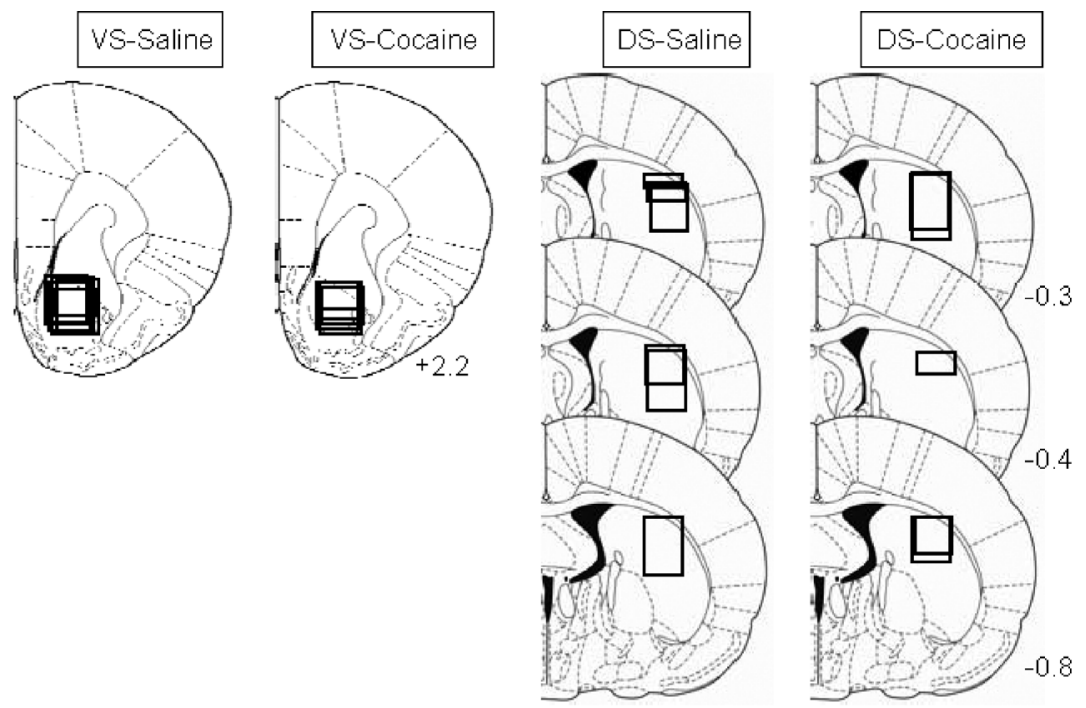

B
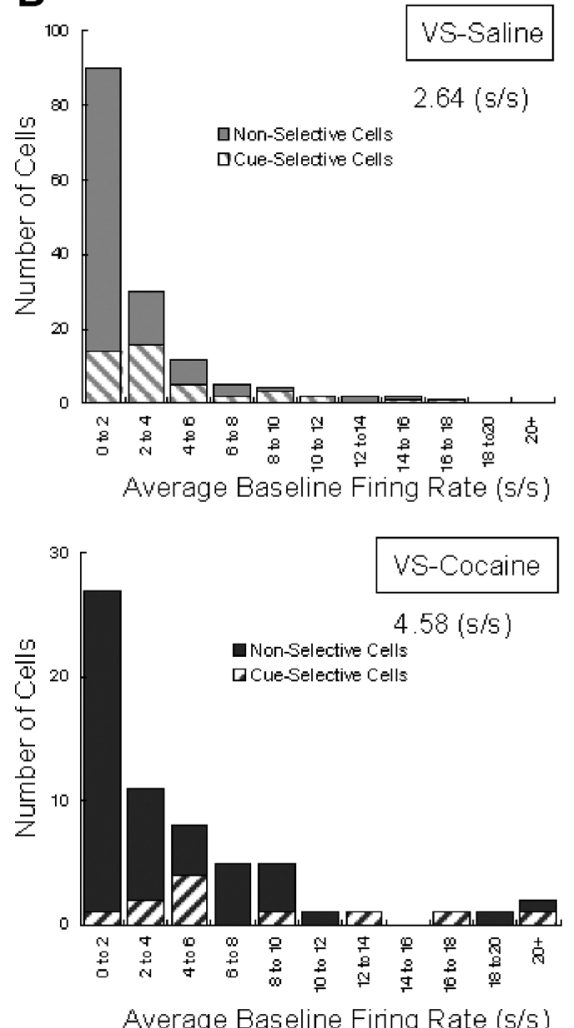
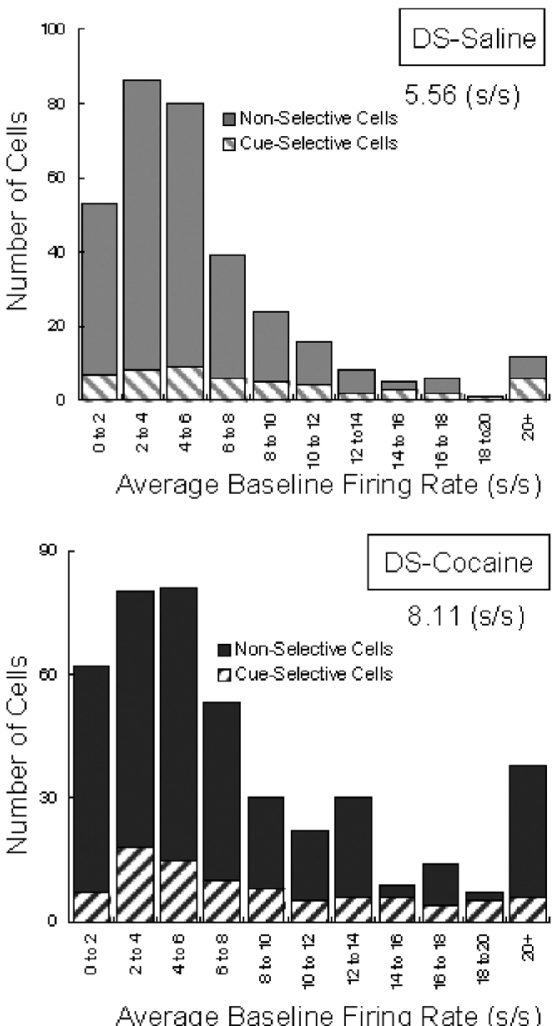

Figure 1. Location of recording sites and distribution of baseline firing rates for VS and DS neurons recorded in saline- and cocaine-treated rats. (A) Recording sites in VS and DS; boxes indicate approximate location of recordings in each rat. (B) Average baseline firing rate and distribution of baseline firing rates for neurons recorded in each group.

\section{Cue-selective activity (or lack thereof) in DS during odor discrimination learning and reversal}

DS-particularly the lateral region-is thought to be important for habit learning mediated by stimulus-response associations (Jog et al., 1999; Frank and Claus, 2006; O'Doherty et al., 2004; Yin et al., 2004; Yin and Knowlton, 2006). Thus DS neurons may fire differently during sampling of cues that are associated with different responses. Accordingly, we found that $15 \%$ (47 out of 318 ) of the neurons recorded in lateral part of DS fired differentially during sampling of the odor cues after learning; these correlates developed with learning and tended to change across reversal in the majority of these neurons (Figure 2 and Table 1). Interestingly even though the vast majority of the cue-selective DS neurons fired more to the positive odor cue (Figure 2 and Table 1), the influence of activity in these neurons on the population response from lateral DS was fairly weak, especially when compared to cue-evoked activity in VS (Figure 3A). Nevertheless the odor-selectivity indices of these neurons, shown in Figure 4B, exhibited a highly significant inverse correlation across reversal, indicating that a subpopulation of DS neurons did track the associative significance of the odor cues across reversal.

Cue-selective activity in DS differed from cue-selective activity in VS in several ways that seemed to reflect the importance of differential responding. For example, the majority of the cue-selective DS neurons 
Table 1. Neural selectivity during cue-sampling during learning and reversal.

\begin{tabular}{|c|c|c|c|c|c|c|c|c|}
\hline \multirow{2}{*}{$\begin{array}{l}\text { Brain region } \\
\text { Outcome in initial learning }\end{array}$} & \multicolumn{2}{|c|}{$\begin{array}{l}\text { VS-saline } \\
(n=148)\end{array}$} & \multicolumn{2}{|c|}{$\begin{array}{c}\text { VS-cocaine } \\
(n=60)\end{array}$} & \multicolumn{2}{|c|}{$\begin{array}{l}\text { DS-saline } \\
(n=318)\end{array}$} & \multicolumn{2}{|c|}{$\begin{array}{c}\text { DS-cocaine } \\
(n=389)\end{array}$} \\
\hline & $\begin{array}{l}\text { Sucrose } \\
\text { odor }\end{array}$ & $\begin{array}{l}\text { Quinine } \\
\text { odor }\end{array}$ & $\begin{array}{c}\text { Sucrose } \\
\text { odor }\end{array}$ & $\begin{array}{l}\text { Quinine } \\
\text { odor }\end{array}$ & $\begin{array}{c}\text { Sucrose } \\
\text { odor }\end{array}$ & $\begin{array}{l}\text { Quinine } \\
\text { odor }\end{array}$ & $\begin{array}{c}\text { Sucrose } \\
\text { odor }\end{array}$ & $\begin{array}{l}\text { Quinine } \\
\text { odor }\end{array}$ \\
\hline Post-criterion (before reversal) & 5 & 39 & 3 & 7 & 29 & 18 & 63 & 22 \\
\hline Pre/Post-criterion (before reversal) & $2 / 5$ & $22 / 39$ & $2 / 3$ & $2 / 7$ & $9 / 29$ & $5 / 18$ & $12 / 63$ & $6 / 22$ \\
\hline $\begin{array}{l}\text { Post-criterion-reversal } \\
\text { (before and after reversal) }\end{array}$ & $0 / 5$ & $24 / 39$ & $2 / 3$ & $2 / 7$ & $11 / 29$ & $3 / 18$ & $20 / 63$ & $5 / 22$ \\
\hline $\begin{array}{l}\text { Post-criterion (before reversal) } \\
+ \text { Pre/Post-criterion (after reversal) }\end{array}$ & na & $18 / 24$ & $2 / 2$ & $2 / 2$ & $3 / 11$ & $3 / 3$ & $6 / 20$ & $3 / 5$ \\
\hline
\end{tabular}

(62\%; 29/47) fired more strongly to the positive odor (Figure 2 and Table 1). Indeed the population of neurons selective for negative cue was not significantly different from chance $\left(X_{2}=0.12, p=0.72\right)$. There was no significant difference between subjects in the proportion of positive and negative cue-selective and non-selective neurons (ANOVA, $F_{2,5}=1.02$, $p=0.45$ ). The predominance of activity to the positive odor cue is evident in the population responses in Figure 4A, which show weakly elevated activity to the positive odor cue both before and after reversal, and in Figure 4B, where a subpopulation of the cue selective neurons tracked the positive odor cue across reversal.

This contrasts sharply with results in VS, where neurons typically fired more to the negative odor cue (Table 1; Figures 2; 3A and 3B). The predominance of firing to the positive odor cue in DS may reflect the difference between the go and no-go responses, rather than some intrinsic difference in encoding of reward and punishment in DS. Since the rewarded go response is highly constrained and the no-go response is not (i.e., the rat is free to execute any number of responses on a no-go), it would be easier to detect neural correlates of the go response. In an area encoding stimulus-response associations, this would bias towards the detection of firing to cues associated with the go response.

Consistent with this speculation, DS neurons that fired to the positive odor cue typically developed cue-selective firing only after rats acquired the behavioral criterion. Of the 29 positive cue-selective DS neurons, $69 \%$ (20/29) of them only developed selectivity post-criterion (Figure 2 and Table 1), a proportion which is significantly higher than the negative cueselective neurons in VS $\left(X_{2}=4.32, p=0.04\right)$. Accordingly the population response in Figure 4A shows no evidence of selectivity pre-criterion. Furthermore, 8 of the $11(73 \%)$ neurons that reversed their cue-selectivity after reversal did so only after rats met reversal criterion (Figure 2 and Table 1), a proportion which is also significantly higher than that in VS $\left(X_{2}=7.12, p=0.008\right)$.

\section{Previous cocaine treatment abolishes cue-selective activity in VS while marginally enhancing cue-selective activity in DS} To examine whether associative encoding in VS and DS was affected by prior cocaine treatment, we compared neural activity during cue sampling in saline- and cocaine-treated rats during learning and across reversal of the odor-outcome associations. The cocaine-treated rats had substantially fewer cue-selective neurons in VS than saline controls after learning (Figure 2 and Table 1), amounting to only $17 \%$ (10 out of 60 neurons) of the population $\left(X_{2}=3.79, p=0.05\right)$. The decline was largely due to the loss of neurons selective for the quinine-predicting odor cue compared to saline controls $\left(X_{2}=5.34, p=0.02\right)$. There was no significant difference between subjects in the proportion of positive and negative cue-selective and non-selective neurons (ANOVA, $F_{2,3}=0.93, p=0.47$ ). The loss of these neurons in cocaine-treated rats was evident in the population responses, illustrated in Figure $3 \mathrm{~A}$, which did not exhibit cue-selectivity in any phase of training. Population responses in VS in cocaine-treated rats
(Figure 3A) also differed from those in saline-treated rats in that there appeared to be a higher baseline firing rate and a general though nonsignificant decline in firing at the time of cue-sampling. In the cueselectivity indices, illustrated in Figure 3B, there was no correlation across reversal in the cocaine-treated group. Indeed, even those neurons that did show selectivity for the quinine-predicting odor cue before reversal were less likely to reverse cue-selectivity. This is evident in Figure 2, in a comparison of the number of single-units that reversed in cocaine versus saline treated rats, and also in Figure 3, which shows that the cue preference in these neurons was not correlated across reversal.

By contrast, cocaine treatment caused a small but significant increase in the responsiveness of DS neurons to the positive odor cue. There was no significant difference between subjects in the proportion of positive and negative cue-selective and non-selective neurons (ANOVA, $F_{2,4}=1.33$, $p=0.33$ ). The increase in firing to the positive cue was evident in a single-unit analysis in which 63 neurons fired more strongly to positive cue (Figure 2 and Table 1). This proportion is significantly larger than that in controls $\left(X_{2}=7.74, p=0.005\right)$. The effect is also somewhat evident in the population responses of the DS neurons in Figure 4A. The relatively weak response to the positive odor cue seen in controls is more robust in the cocaine-treated rats. The stronger response to the positive odor in cocaine-treated rats was particularly evident in the pre-criterion trials, though this difference was not borne out at the single-unit level (Figure 2 and Table 1). Furthermore the correlation in the odor-selectivity on a unitby-unit basis, shown in Figure 4B, was largely unchanged, indicating that the flexibility of encoding in these neurons was not altered.

\section{DISCUSSION}

In this study, we compared cue-selective activity in VS and DS in rats with and without a history of cocaine exposure. Rats received saline or cocaine for 14 days and then neural activity was recorded from VS and DS during acquisition and reversal of a series of 2-odor go, no-go discriminations. In the saline-treated controls, neurons in both VS and DS became cueselective. However, there were several differences in cue-selectivity between these two regions that seemed to reflect their presumed involvement in different associative learning functions. Specifically cueselective activity in DS seemed to be more directly related to associations between the cues and the stereotyped go-response, whereas cueselective activity in VS seemed to be less directly related to discriminative responding and more reflective of the valence or motivational significance of the cue. Cocaine shifted the balance of associative encoding between these two regions, abolishing the strong cue-selectivity normally present in VS while marginally enhancing the relatively weak cue-selectivity normally present in DS. Thus cocaine exposure causes regionally specific effects on neural processing in striatum; these effects may promote the habitization of behavior by shifting control from ventral to dorsolateral regions. 
A A Pre/Post-Criterion
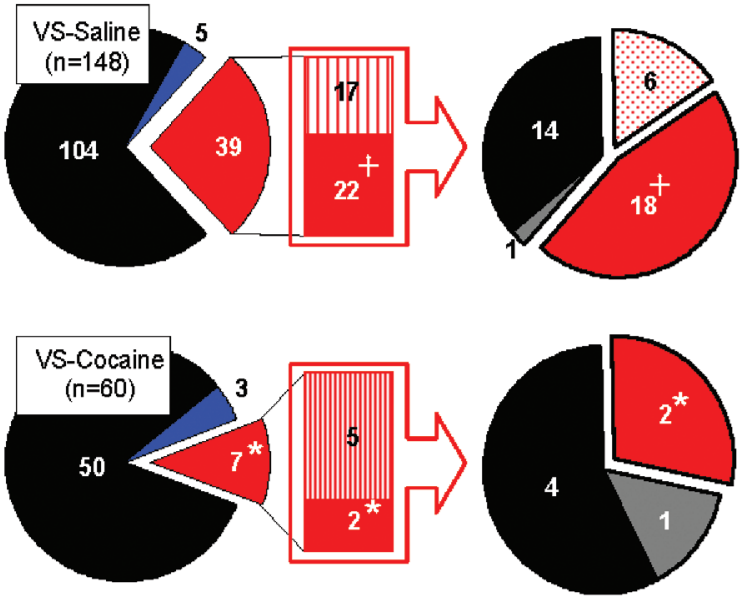

Non-Selective

Sucrose Cue-Selective

Quinine Cue-Selective

III (Post-Criterion only)

Quinine Cue-Selective

(Pre/Post-Criterion)

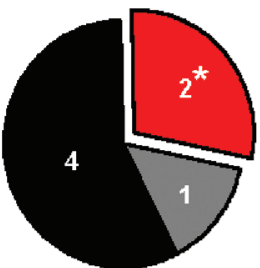

Reversal

Non-Selective

Same Odor

Reversed Cue-Preference

(Post-Criterion only)

Reversed Cue-Preference (Pre/Post-Criterion)
Pre/Post-Criterion

Reversal
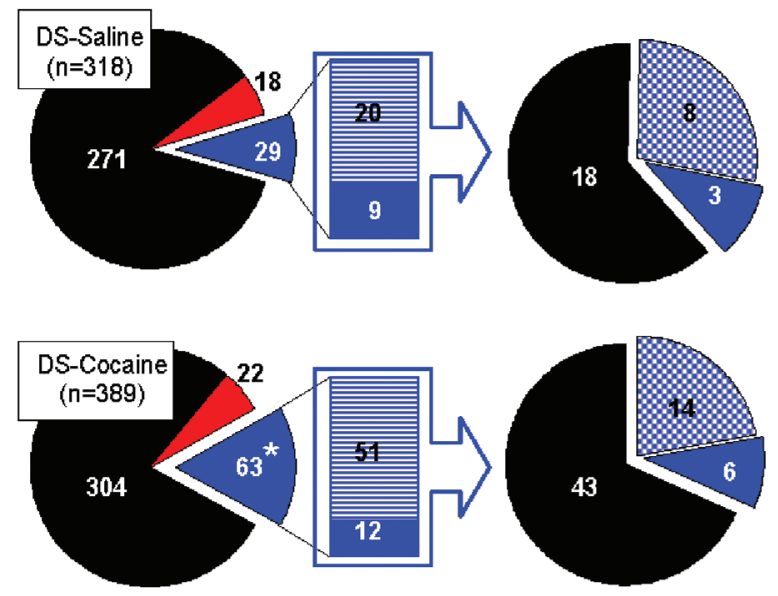

Non-Selective

Quinine Cue-Selective

Sucrose Cue-Selective

$\boxminus$ (Post-Criterion only)

Sucrose Cue-Selective

(Pre/Post-Criterion)
Non-Selective

Reversed Cue-Preference

8 (Post-Criterion only)

Reversed Cue-Preference (Pre/Post-Criterion)

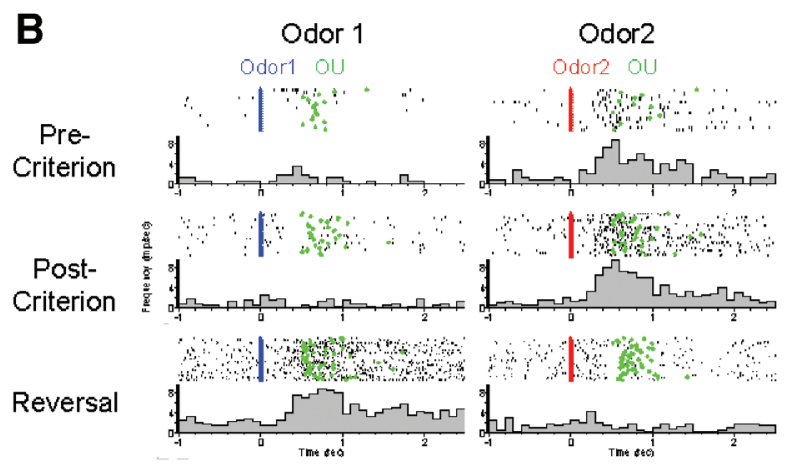

Figure 2. Populations of cue-selective neurons of VS and DS recorded in saline- and cocaine-treated rats during discrimination and reversal learning. (A) Pie-charts in left side of VS-saline and VS-cocaine groups indicate populations of neurons which show non-selective (black), sucrose cue-selective (blue), and quinine cue-selective (red) firing. Bar-graphs in the middle of VS-saline and VS-cocaine groups indicate population of neurons which show quinine cue-selectivity after post-criterion trials (red stripe) and quinine cue-selectivity in both pre-and post-criterion trials (red). Pie-charts in right side of VS-saline and VS-cocaine groups indicate changes of cue-selectivity of quinine cue-selective VS neurons after reversal. Black indicates neurons which are non-selective after reversal. Red mesh indicates VS neurons which develop quinine cue-selectivity in post-criterion trials of reversal learning. Red indicates VS neurons which show quinine cueselectivity in both pre- and post-criterion trials of reversal learning. Gray indicates VS neurons which maintained the same cue-selectivity after reversal. Pie-charts in left side of DS-saline and DS-cocaine groups indicate populations of neurons which show non-selective (black), sucrose cue-selective (blue), and quinine cueselective (red) firing. Bar-graphs in the middle of DS-saline and DS-cocaine groups indicate population of neurons which show sucrose cue-selectivity after postcriterion trials (blue stripe) and sucrose cue-selectivity in both pre- and post-criterion trials (blue). Pie-charts in right side of DS-saline and DS-cocaine groups indicate changes of cue-selectivity of sucrose cue-selective DS neurons after reversal. Black indicates neurons which are non-selective after reversal. Blue mesh indicates DS neurons which develop sucrose cue-selectivity in post-criterion trials of reversal learning. Blue indicates DS neurons which show sucrose cueselectivity in both pre- and post-criterion trials of reversal learning ${ }^{*}$, significant difference from saline group at $p<0.05$ or better; ${ }^{+}$, significant difference between VS and DS at $p<0.05$ or better). (B) Examples of a negative cue-selective VS neuron in saline-treated controls and a positive cue-selective DS neuron in cocaine-treated rats during pre-criterion, post-criterion and reversal trial. Activity is shown synchronized to the onset of odor1 (Blue) or odor2 (Red). Activity is displayed in raster format at the top and as a peri-event time histogram in spikes/second in $100 \mathrm{~ms}$ bin at the bottom of each panel. Green indicates nose-unpoke from the odor port.

\section{Cue-selective activity in DS and VS reflects different associative learning functions}

One perhaps unexpected aspect of our results is that neurons in VS and DS were more responsive to cues associated with aversive and appetitive outcomes, respectively. Neurons in VS developed strong selectivity for the odor cue that predicted aversive quinine solution; only a small number of VS neurons developed cue-selectivity for the positive odor cue. By contrast, DS neurons tended to become selective for the positive odor cue, which predicted sucrose. We do not believe this reflects the differential involvement of these striatal regions in appetitive and aversive learning. 

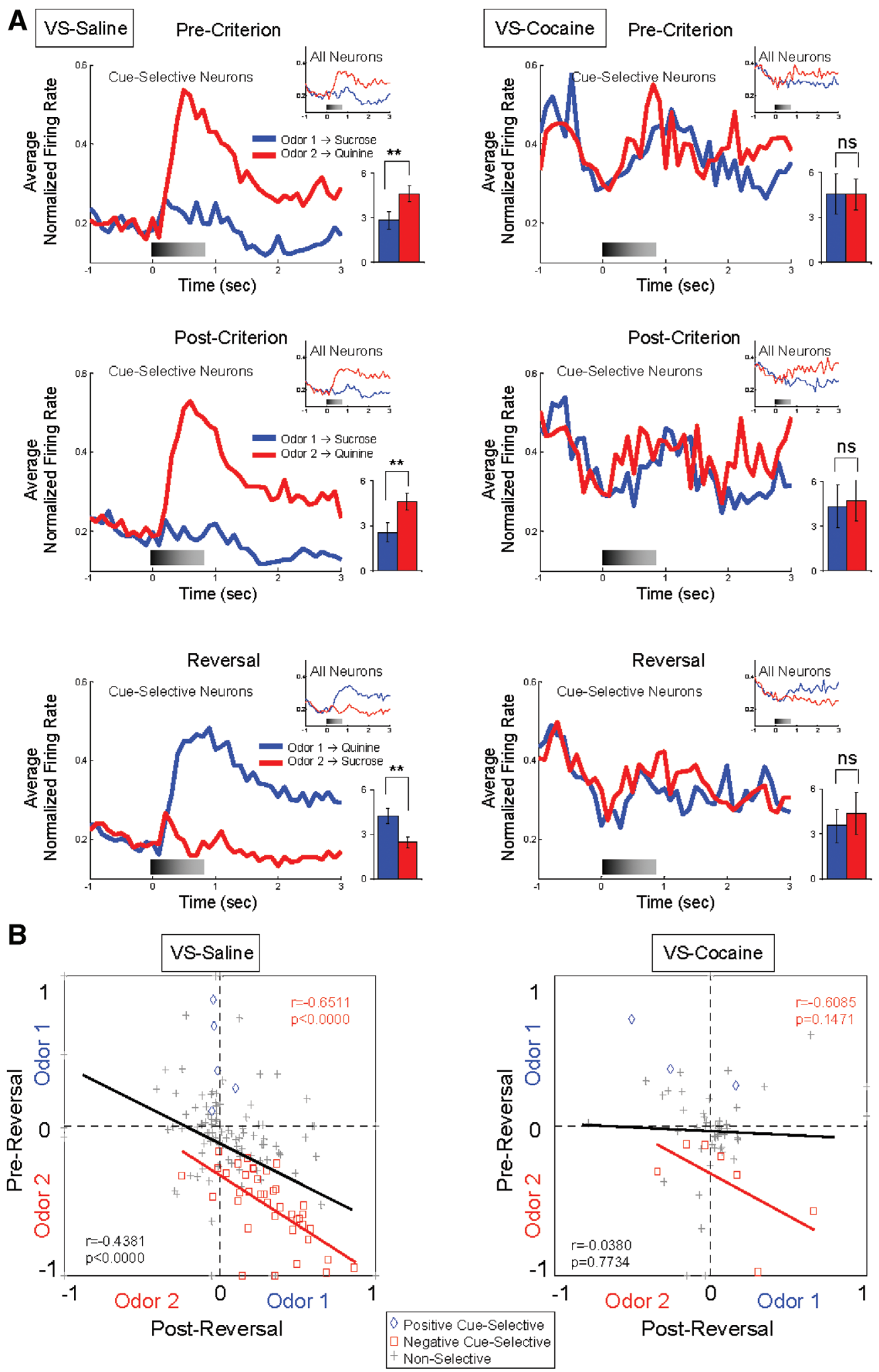

Figure 3. Cue-selectivity in VS neurons recorded in saline- and cocaine-treated rats during discrimination and reversal learning. (A) Population responses to each odor cue are shown for cue-selective VS neurons and all VS neurons (small inset) during pre-criterion trials, post-criterion trials and postreversal. Shown are average normalized firing rates, in spikes/second in $100 \mathrm{~ms}$ bins, for odor 1 (Blue) and odor 2 (Red) trials. Gray shading in each histogram indicates the approximate timing of odor sampling. Bar graph within each histogram shows averaged firing rates of odor 1 (blue) and odor 2 (red) sampling phases ${ }^{*}$, significant difference at $p<0.05$; **, significant difference at $p<0.01$ or better, ANOVA). The neural population in saline-treated controls exhibited strong phasic firing to the odor cue that predicted the aversive quinine outcome, in pre-criterion trials, post-criterion trials, and after reversal. In contrast, the neural population in cocaine-treated rats was not cue-selective in either phase. (B) Odor-selectivity indices during odor sampling before and after reversal for VS neurons recorded in saline- and cocaine-treated rats. Indices were calculated for each neuron as ( $\left.f r_{\text {odor1 }}-f r_{\text {odor } 2}\right) /\left(\left(f r_{\text {odor } 1}+f r_{\text {odor }}\right)\right.$, where $f r_{\text {odor1 }}$ was the firing rate during sampling of the odor that predicted the sucrose outcome in pre-reversal trials and $\mathrm{fr}_{\text {odor }}$ was the firing rate during sampling of the odor that predicted the quinine outcome in pre-reversal trials. Blue diamonds and red squares denote neurons that were selective before reversal for odor 1 or odor 2, respectively. Gray crosses denote neurons that were non-selective. Black line indicates correlation of all neurons and red line indicates correlation of negative cue-selective neurons. 

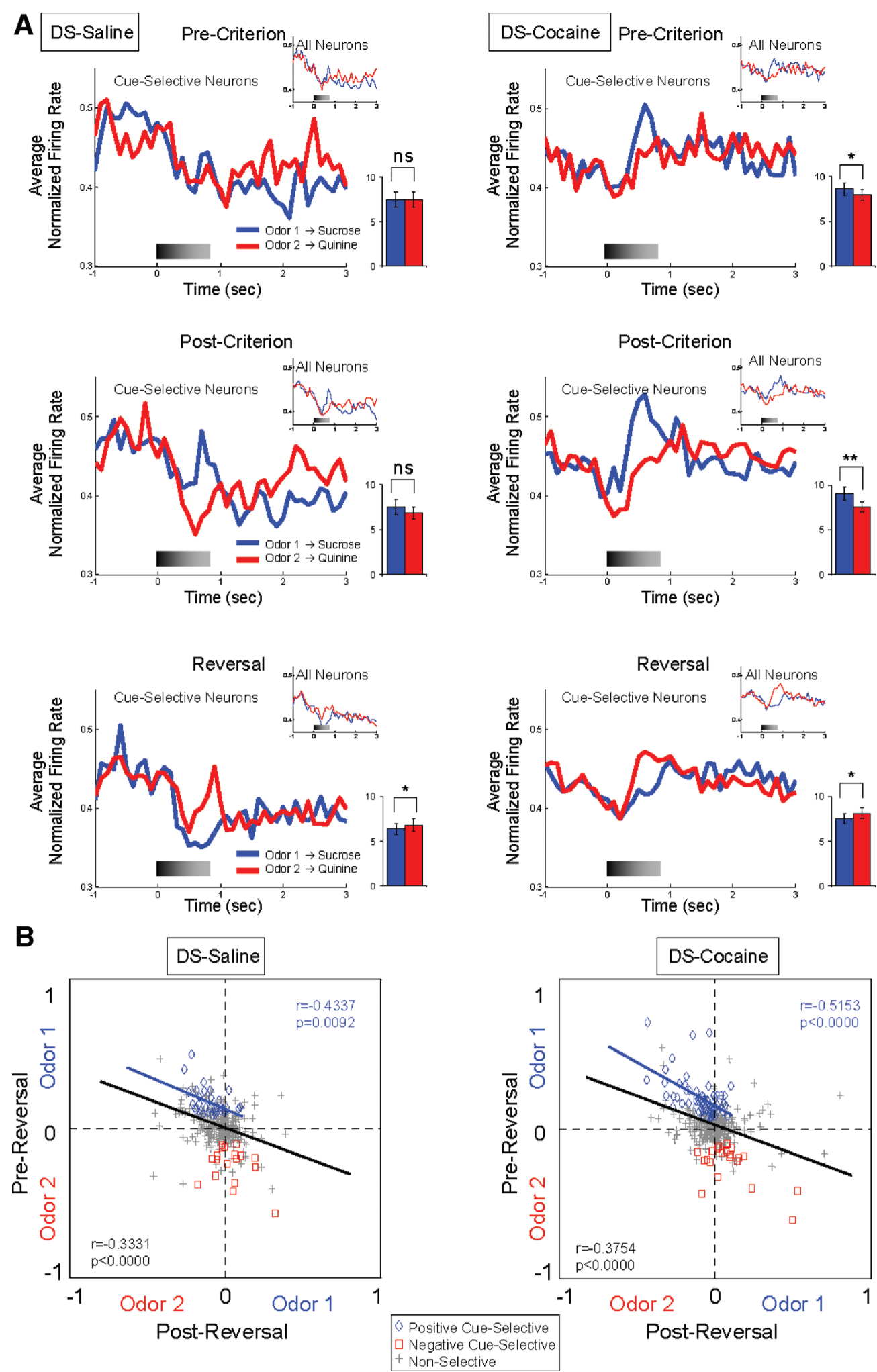

Figure 4. Cue-selectivity in DS neurons recorded in saline- and cocaine-treated rats during discrimination and reversal learning. (A) Population responses to each odor cue are shown for cue-selective DS neurons and all DS neurons (small inset) during pre-criterion trials, post-criterion trials and postreversal. Shown are average normalized firing rates, in spikes/second in $100 \mathrm{~ms}$ bins, for odor 1 (Blue) and odor 2 (Red) trials. Gray shading in each histogram indicates the approximate timing of odor sampling. Bar graph within each histogram shows averaged firing rates of odor 1 (blue) and odor 2 (red) sampling phases ${ }^{*}$, significant difference at $p<0.05{ }^{* *}$, significant difference at $p<0.01$ or better, ANOVA). The neural population in cocaine-treated group exhibited stronger phasic firing to the odor cue that predicted the sucrose outcome than that in control group, especially pre-criterion and post-criterion trials. (B) Odor-selectivity indices during odor sampling before and after reversal for DS neurons recorded in saline- and cocaine-treated rats. Indices were calculated for each neuron as $\left(f r_{o d o r 1}-f r_{\text {odor } 2}\right) /\left(f r_{\text {odor1 }}+f r_{\text {odor } 2}\right)$, where $f r_{\text {odort }}$ was the firing rate during sampling of the odor that predicted the sucrose outcome in pre-reversal trials and fr $r_{\text {odor } 2}$ was the firing rate during sampling of the odor that predicted the quinine outcome in pre-reversal trials. Blue diamonds and red squares denote neurons that were selective before reversal for odor 1 or odor 2, respectively. Gray crosses denote neurons that were non-selective. Black line indicates correlation of all neurons and blue line indicates correlation of positive cue-selective neurons. 
Instead we would speculate that it reflects the unique aspects of our task and the respective roles of VS and DS in processing different types of associative information.

For example, the quinine solution we used is likely more motivationally significant or intense than our dilute sucrose solution. The predominance of neurons in VS that were selective for the quinine-predicting cue would be consistent with the proposal that VS neurons encode the general affective or motivational significance that cues acquire through pairing with primary reinforcers (O'Doherty et al., 2004; Parkinson et al., 1999). It is also consistent with this idea that most of the cue-selective neurons in VS developed differential firing before the rats exhibited accurate choice performance during initial learning and also after reversal.

Similarly the predominance of neurons in DS that were selective for the sucrose-predicting cue would be consistent with the proposal that DS neurons encode associations between cues and subsequent responses (Frank and Claus, 2006; Jog et al., 1999; 0'Doherty et al., 2004; Yin and Knowlton, 2006; Yin et al., 2004). This is because the go response associated with the sucrose-predicting cue is much more constrained than the no-go response associated with the quinine-predicting cue (i.e., the rat is free to execute any number of responses when it does not go to the well). It is easier to detect neural correlates of a highly constrained, stereotyped response. Accordingly, most of the cue-selective DS neurons developed differential firing only after the rats were performing accurately on the initial or the reversed discrimination.

\section{Cocaine shifts the balance of associative encoding from ventral to dorsolateral striatum}

Repeated exposure to psychostimulants has enduring effects on the structure and function of brain areas that play important roles in learning and memory (Everitt and Wolf, 2002). These changes are proposed to promote habitual behavior, evident in over-responding in behavioral settings that are specifically linked to striatal function. For example, instrumental behavior in drug-naïve rats becomes resistant to outcome devaluation with extended training. This phenomenon, which is dependent on lateral DS (Yin et al., 2004), is interpreted as reflecting the development of motor habits mediated by cue-response associations. Exposure to psychostimulants causes instrumental responding to become resistant to devaluation even without overtraining (Nelson and Killcross, 2006). Similarly exposure to psychostimulants causes instrumental responding to become more sensitive to the motivating effects of Pavlovian cues (Wyvell and Berridge, 2001); this transfer effect is dependent on VS (Corbit et al., 2001; Hall et al., 2001). More rapid development of motor habits and increased transfer could reflect a general drug-induced increase in the efficacy of cue-evoked associative processing in DS and VS.

Our results are only partially consistent with this proposal. Specifically rather than causing a general increase in the efficacy of neural processing in both areas, cocaine exposure caused a lasting shift in the balance of associative encoding away from VS and towards DS. This shift largely reflected a reduction in the degree and also flexibility of cue-selective firing in VS. Nevertheless it is worth noting that DS is unique among the brain regions where we have recorded in cocaine-treated rats in that it largely maintained or even enhanced its normal pattern of cue-evoked activity (Stalnaker et al., 2006; Stalnaker et al., 2007b).

Stability of encoding in DS combined with disrupted encoding in corticolimbic circuits-orbitofrontal cortex, basolateral amygdala, and VS-might cause simple motor habits to become somewhat more influential over behavior and motivational habits to become less influential. Clearly this might explain evidence that exposure to psychostimulants increases the rate at which instrumental responding becomes resistant to reinforcer devaluation (Nelson and Killcross, 2006). However, the relatively weak changes in DS suggest that the primary cause of this behavioral change is not enhanced processing in DS but rather must decline in other parts of the circuit governing goal-directed behavior-such as prefrontal areas or perhaps medial part of DS (Corbit and Balleine, 2003; Hitchcott et al., 2007; Yin et al., 2005a; Yin et al., 2005b).
Perhaps more interesting is the finding that VS neurons in cocainetreated rats were clearly less responsive to the significance of the cues. Several features of our study may account for this result. For example, as noted earlier, our task uses a strongly aversive outcome-quinine-to motivate behavior. While neurons in some brain regions are equally responsive on positive and negative trials, activity in VS in this and an earlier study (Setlow et al., 2003) seems to be more sensitive to this highly motivating, more intense, aversive outcome. It may be that effects of drug exposure on processing of appetitive information in VS were overwhelmed in our task by the presence of quinine. This explanation would reconcile our results with evidence that cocaine exposure causes enhanced transfer (Wyvell and Berridge, 2001), thought to reflect representations of affective significance stored in VS (Corbit et al., 2001; Hall et al., 2001), and also with recent evidence that drug-associated cues are strongly represented in VS, particularly in accumbens core (Hollander and Carelli, 2007).

On the other hand, enhanced transfer may reflect drug-induced increases in the responsiveness of other brain regions to associative information. For example, we have found enduring changes in cueselective firing in basolateral amygdala in cocaine-treated rats (Stalnaker et al., 2007b). Neurons in drug-treated rats were more responsive to the appetitive odor cues, firing in greater numbers to the positive odor cue earlier in learning and persisting in firing to this cue even after reversal. Amygdala is also implicated in Pavlovian-to-instrumental transfer (Corbit and Balleine, 2005; Holland and Gallagher, 2003). Alternatively transfer has been reported to increase with over-training (Holland, 2004), thus effects of cocaine might reflect a general increase in the rate of habit formation, due to enhanced processing in lateral DS. Indeed there is a report that lateral DS is important for transfer (Corbit et al., 2007). Thus the effect of drug exposure on transfer may reflect processing changes in lateral DS or amygdala rather than VS.

A second feature of our study that may account for our findings in VS is that we tested the effects of passive drug-exposure on encoding of nondrug cues. It may be that self-administration of addictive drugs causes enhanced processing in VS but passive exposure does not, or that changes in VS are specific to drugs and drug-associated cues. As noted above, drug-associated cues are effective at activating VS neurons, particularly after prolonged abstinence from cocaine (Hollander and Carelli, 2007). Indeed it has been proposed that addiction reflects enhanced processing of drug-related cues at the expense of other non-drug information (Kalivas and Volkow, 2005; Kalivas and Hu, 2006). The absence of cue-selective firing in VS in our task may reflect a decline in responsiveness of this circuit that is specific to non-drug cues.

\section{CONFLICT OF INTEREST STATEMENT}

The authors declare that the research was conducted in the absence of any commercial or financial relationships that could be construed as a potential conflict of interest.

\section{ACKNOWLEDGMENTS}

This work was supported by a Long-Term Fellowship from the Human Frontiers Science Program to YT and by R01-DA015718 to GS from the NIDA. M.R. and T.S. were supported by training grants from NINDS (T32NS07375 and NIDCD (T32-DC00054), respectively. The authors would like to thank Dr. Stephen Warrenburg at International Flavors and Fragrances for his assistance obtaining odor compounds.

\section{REFERENCES}

Burke, K. A., Franz, T. M., Gugsa, N., and Schoenbaum, G. (2006). Prior cocaine exposure disrupts extinction of fear conditioning. Learn. Mem. 13, 416-421.

Carelli, R. M. (2002). Nucleus accumbens cell firing during goal-directed behaviors for cocaine vs. 'natural' reinforcement. Physiol. Behav. 76, 379-387.

Corbit, L. H., and Balleine, B. W. (2003). The role of prelimbic cortex in instrumental conditioning. Behav. Brain. Res. 146, 145-157.

Corbit, L. H., and Balleine, B. W. (2005). Double dissociation of basolateral and central amygdala lesions on the general and outcome-specific forms of pavlovianinstrumental transfer. J. Neurosci. 25, 962-970. 
Corbit, L. H., Muir, J. L., and Balleine, B. W. (2001). The role of the nucleus accumbens in instrumental conditioning: evidence of a functional dissociation between accumbens core and shell. J. Neurosci. 21, 3251-3260.

Corbit, L. H., and Janak, P. H. (2007). Inactivation of the lateral but not medial dorsal striatum eliminates the excitatory impact of Pavlovian stimuli on instrumental responding. J. Neurosci. 27, 13977-13981.

Everitt, B. J., and Wolf, M. E. (2002). Psychomotor stimulant addiction: a neural systems perspective. J. Neurosci. 22, 3312-3320.

Everitt, B. J., and Robbins, T. W. (2005). Neural systems of reinforcement for drug addiction: from actions to habits to compulsion. Nat. Neurosci. 8, 1481-1489.

Frank, M. J., and Claus, E. D. (2006). Anatomy of a decision: striato-orbitofrontal interactions in reinforcement learning, decision making, and reversal. Psychol. Rev. 113, 300-326.

Hall, J., Parkinson, J. A., Connor, T. M., Dickinson, A., and Everitt, B. J. (2001) Involvement of the central nucleus of the amygdala and nucleus accumbens core in mediating Pavlovian influences on instrumental behaviour. Eur. J. Neurosci. 13, 1984-1992.

Hitchcott, P. K., Quinn, J. J., and Taylor, J. R. (2007). Bidirectional modulation of goal-directed actions by prefrontal cortical dopamine. Cereb. Cortex. 17, 28202827.

Holland, P. C. (2004). Relations between Pavlovian-Instrumental transfer and reinforcer devaluation. J. Exp. Psychol.: Anim. Behav. Proc. 30, 104-117.

Holland, P. C., and Gallagher, M. (2003). Double dissociation of the effects of lesions of basolateral and central amygdala on conditioned stimulus-potentiated feeding and Pavlovian-instrumental transfer. Eur. J. Neurosci. 17, 1680-1694.

Hollander, J. A., and Carelli, R. M. (2007). Cocaine-associated stimuli increase cocaine seeking and activate accumbens core neurons after abstinence. J. Neurosci. 27 3535-3539.

Homayoun, H., and Moghaddam, B. (2006). Progression of cellular adaptations in medial prefrontal and orbitofrontal cortex in response to repeated amphetamine. $J$. Neurosci. 26, 8025-8039.

Jog, M. S., Kubota, Y., Connolly, C. I., Hillegaart, V., and Graybiel, A. M. (1999). Building neural representations of habits. Science 286, 1745-1749.

Kalivas, P. W., and Volkow, N. D. (2005). The neural basis of addiction: a pathology of motivation and choice. Am. J. Psychiatr. 162, 1403-1413.

Kalivas, P. W., and Hu, X. T. (2006). Exciting inhibition in psychostimulant addiction. Trends Neurosci. 29, 610-616.

Nelson, A., and Killcross, S. (2006). Amphetamine exposure enhances habit formation. J. Neurosci. 26, 3805-3812.

Nicola, S. M., Yun, I. A., Wakabayashi, K. T., and Fields, H. L. (2004). Cue-evoked firing of nucleus accumbens neurons encodes motivational significance during a discriminative stimulus task. J. Neurophysiol. 91, 1840-1865.

O'Doherty, J., Dayan, P., Schultz, J., Deichmann, R., Friston, K., and Dolan, R. J. (2004). Dissociable roles of ventral and dorsal striatum in instrumental conditioning. Science 304, 452-454.

Parkinson, J. A., Robbins, T. W., and Everitt, B. J. (1999). Selective excitotoxic lesions of the nucleus accumbens core and shell differentially affect aversive Pavlovian conditioning to discrete and contextual cues. Psychobiology 27, 256-266.
Roitman, M. F., Wheeler, R. A., and Carelli, R. M. (2005). Nucleus accumbens neurons are innately tuned for rewarding and aversive taste stimuli, encode their predictors, and are linked to motor output. Neuron 45, 587-597.

Schmitzer-Torbert, N., and Redish, A. D. (2004). Neuronal activity in the rodent dorsal striatum in sequential navigation: separation of spatial and reward responses on the multiple T task. J. Neurophysiol. 91, 2259-2272.

Schoenbaum, G., and Setlow, B. (2005). Cocaine makes actions insensitive to outcomes but not extinction: implications for altered orbitofrontal-amygdalar function. Cereb. Cortex 15, 1162-1169.

Schoenbaum, G., Chiba, A. A., and Gallagher, M. (1999). Neural encoding in orbitofrontal cortex and basolateral amygdala during olfactory discrimination learning. $J$. Neurosci. 19, 1876-1884.

Schoenbaum, G., Saddoris, M. P., Ramus, S. J., Shaham, Y., and Setlow, B. (2004). Cocaine-experienced rats exhibit learning deficits in a task sensitive to orbitofrontal cortex lesions. Eur. J. Neurosci. 19, 1997-2002.

Schultz, W., Tremblay, L., and Hollerman, J. R. (2000). Reward processing in primate orbitofrontal cortex and basal ganglia. Cereb. Cortex 10, 272-284.

Setlow, B., Schoenbaum, G., and Gallagher, M. (2003). Neural encoding in ventral striatum during olfactory discrimination learning. Neuron $38,625-636$.

Shidara, M., Aigner, T. G., and Richmond, B. J. (1998). Neuronal signals in the monkey ventral striatum related to progress through a predictable series of trials. J. Neurosci. $18,2613-2625$.

Stalnaker, T. A., Franz, T. M., Singh, T., and Schoenbaum, G. (2007a). Basolateral amygdala lesions abolish orbitofrontal-dependent reversal impairments. Neuron 54 , 51-58.

Stalnaker, T. A., Roesch, M. R., Franz, T. M., Burke, K. A., and Schoenbaum, G. (2006). Abnormal associative encoding in orbitofrontal neurons in cocaine-experienced rats during decision-making. Eur. J. Neurosci. 24, 2643-2653.

Stalnaker, T. A., Roesch, M. R., Franz, T. M., Calu, D. J., Singh, T., and Schoenbaum, G. (2007b). Cocaine-induced decision-making deficits are mediated by miscoding in basolateral amygdala. Nat. Neurosci. 10, 949-951.

Williams, G. V., Rolls, E. T., Leonard, C. M., and Stern, C. (1993). Neuronal responses in the ventral striatum of the behaving macaque. Behav. Brain Res. 55, 243252.

Wyvell, C. L., and Berridge, K. C. (2001). Incentive sensitization by previous amphetamine exposure: increased cue-triggered "wanting" for sucrose reward. J. Neurosci. 21, 7831-7840.

Yin, H. H., and Knowlton, B. J. (2006). The role of the basal ganglia in habit formation. Nat. Rev. Neurosci. 7, 464-476.

Yin, H. H., Knowlton, B. J., and Balleine, B. W. (2004). Lesions of dorsolateral striatum preserve outcome expectancy but disrupt habit formation in instrumental learning. Eur. J. Neurosci. 19, 181-189.

Yin, H. H., Knowlton, B. J., and Balleine, B. W. (2005a). Blockade of NMDA receptors in the dorsomedial striatum prevents action-outcome learning in instrumental conditioning. Eur. J. Neurosci. 22, 505-512.

Yin, H. H., Ostlund, S. B., Knowlton, B. J., and Balleine, B. W. (2005b). The role of the dorsomedial striatum in instrumental conditioning. Eur. J. Neurosci. 22, 513523. 